\title{
Ausgangsbedingungen und Verfahren für das Recycling von Wolframkarbid-Verbundwerkstoffen
}

\author{
Tamara Ebner · Claudia Kerschbaumer · Gregor Kücher · Stefan Luidold • Christian Storf • Christoph Czettl • \\ Thomas Wolfe $\cdot$ Anthony Smith
}

Online publiziert: 5. September 2017

(c) Der/die Autor(en) 2017. Dieser Artikel ist eine Open-Access-Publikation.

Zusammenfassung Das Recycling von WC-Verbunden bietet eine Vielzahl an ökologischen, wirtschaftlichen und strategischen Vorteilen. Da Österreich den gesamten Produktlebenszyklus von Wolfram, dem Ausgangstoff für Wolframkarbid (WC), von Bergbau, Herstellung sowie Aufarbeitung der anfallenden sekundären Materialen abdeckt, hat diese Thematik einen entsprechend hohen Stellenwert für die inländische Ökonomie. Beim Recycling lässt sich grundsätzlich zwischen direkten, semidirekten und indirekten Verfahrensvarianten unterscheiden, welche zum Teil seit Langem industriell umgesetzt sind und angewandt werden. Dennoch besteht unter anderem hinsichtlich eingeschleppter Verunreinigungen oder neuartiger Bindersysteme erheblicher Forschungs- und Entwicklungsaufwand, weshalb sich das CD-Labor für Extraktive Metallurgie von Technologiemetallen intensiv mit diesen Fragestellungen auseinandersetzt. Grundlegende Untersuchungen der einzelnen Prozessschritte und somit das Schaffen eines detaillierten Verständnisses der relevanten Vorgänge sollen einen wesentlichen Beitrag zur Verbesserung des WC-Verbundrecyclings liefern. Dies er-

DI Dr. T. Ebner $(\bowtie)$.

DI C. Kerschbaumer · DI G. Kücher · Priv.-Doz. DI Dr. S. Luidold

CD-Labor für Extraktive Metallurgie von Technologiemetallen/Lehrstuhl für Nichteisenmetallurgie,

Montanuniversität Leoben,

Franz-Josef-Straße 18, 8700 Leoben,

Österreich

tamara.ebner@unileoben.ac.at

DI C. Storf · DI Dr. C. Czettl

CERATIZIT Austria GmbH,

Metallwerk-Plansee-Straße 71, 6600 Reutte, Österreich

Dr. T. Wolfe $\cdot$ Dr. A. Smith

Global Tungsten \& Powders Corp., 1

Hawes Street, 18848 Towanda, PA, USA möglicht eine Vermeidung zukünftiger Versorgungsengpässe und die Basis zur Verbesserung der Ressourceneffizienz des kritischen Rohstoffes Wolfram.

Schlüsselwörter Wolframkarbid . Hartmetalle · Recycling · Zinkprozess . Laugung

\section{Original conditions and processes for the recycling of tungsten carbide composites}

Abstract The recycling of WC composite materials offers a wide range of advantages in ecological, economic and strategic fields. Since Austria covers the entire product life cycle along the tungsten supply chain, the source material for tungsten carbides (WC), from mining and production to recycling, this topic is of particular importance to the domestic economy. In general, it can be distinguished between direct, semi direct and indirect recovery routes, which are partly implemented and used industrially. Nevertheless these methods still require a significant extent of research and development regarding for instance introduced impurities or novel binder systems. Therefore, the CD Laboratory for Extractive Metallurgy of Technological Metals intensively examines these topics. Fundamental studies concerning the individual process steps create a detailed understanding of the respective operations and contribute to the improvement of the WC composite recycling. This counteracts future supply shortfalls and constitutes furthermore the basis for an increase of resource efficiency of the critical raw material tungsten.

Keywords Tungsten carbide Cemented carbide $\cdot$ Recycling $\cdot$ Zinc process · Leaching

\section{Einleitung}

Im Rahmen des Berichts „Critical raw materials for the EU“ erfolgte im Jahr 2010 eine Bewertung verschiedener Rohstoffe hinsichtlich ihrer wirtschaftlichen Bedeutung als auch ihres Versorgungsrisikos durch die Ad-hoc Working Group (2010). Im Rahmen dieser Veröffentlichung wurde eine Reihe von Materialien als kritisch eingestuft, da sie sowohl von wirtschaftlichen als auch versorgungstechnischen Standpunkten her einen besonderen Stellenwert aufweisen. Im Zuge der Rohstoffstudie von Luidold et al. (2013), welche sich intensiv mit kritischen Substanzen in den österreichischen Hochtechnologieanwendungen auseinandersetzte, fand eine Erweiterung des von der Adhoc-Working-Group erarbeiteten Diagramms um das Verhältnis der nationalen Importe bezogen auf die Weltproduktion statt. Dies lässt eine Bewertung der Bedeutsamkeit der betrachteten Materialien für die österreichische Industrie zu. Die folgende Abb. 1 zeigt, dass Wolfram durch seine hohe wirtschaftliche Relevanz und ein entsprechendes Versorgungsrisiko der Gruppe der kritischen Rohstoffe zuzuordnen ist. Des Weiteren zeichnet sich dieses Refraktärmetall aufgrund des vergleichsweise hohen Verhältnisses von Importen zur W-Weltproduktion von $>1 \%$ durch einen hohen Stellenwert in der österreichischen Ökonomie aus (Ad-hoc Working Group 2010; Luidold et al. 2013).

Darüber hinaus deckt Österreich den gesamten W-Produktlebenszyklus von Bergbau, Produktion und Recycling durch global agierende Betriebe wie die Plansee Gruppe mit Standort in Reutte, Wolfram Bergbau und Hütten AG in Mittersill sowie St. Martin i.S. und Böhlerit in Kapfenberg ab. 


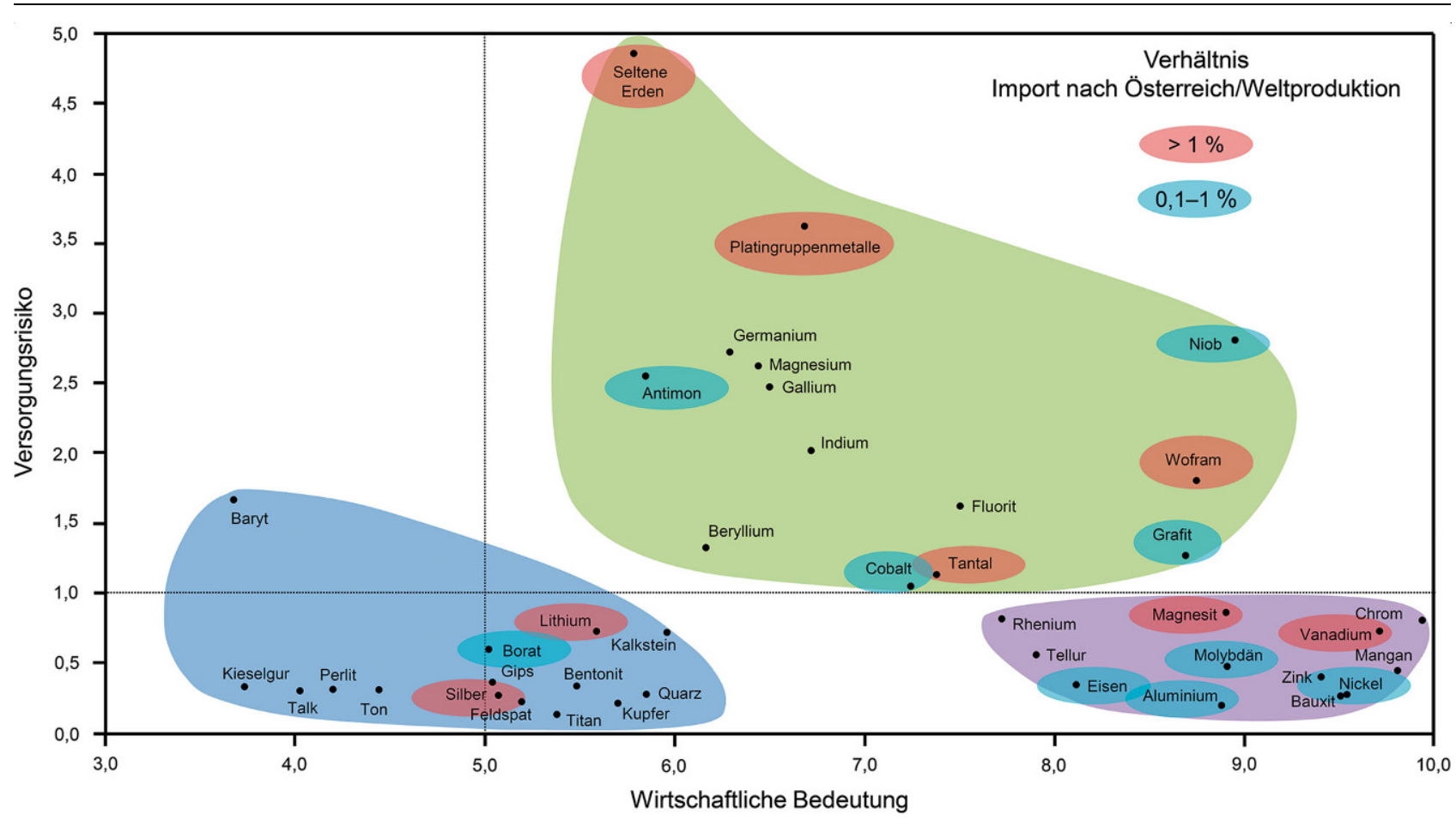

Abb. 1 Relevanz verschiedener Rohstoffe für die Europäische Union abhängig von ihrer wirtschaftlichen Bedeutung und ihrem Versorgungsrisiko unter Berücksichtigung des Verhältnisses von österreichischem Import zur Weltproduktion nach der Ad-hoc Working Group (2010) und Luidold et al. (2013) - Grün hohes Risiko, große Auswirkung; Violett geringes Risiko, große Auswirkung; Blau niedriges Risiko, geringe Auswirkung (Ebner 2016)
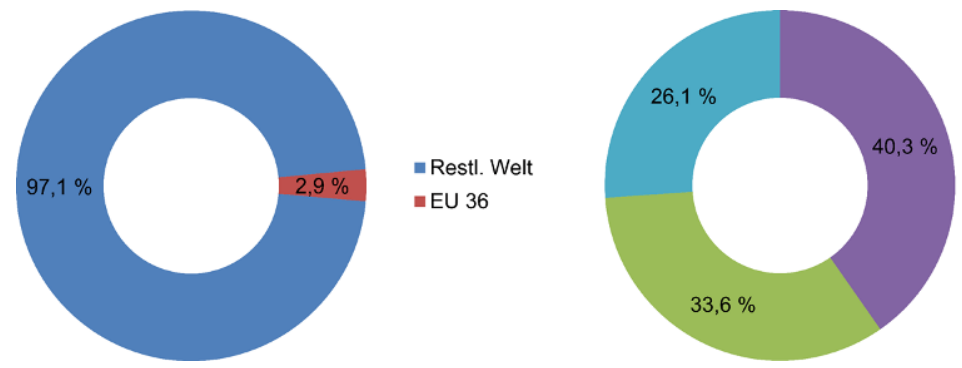

$\because$ Restl. Welt $=\mathrm{EU} 36$

- Spanien

- Österreich = Portugal

Abb. 2 Anteil der W-Bergwerksproduktion der EU 36 an der weltweiten Förderung (links) sowie prozentuelle Verteilung innerhalb der EU im Jahr 2014 (rechts) nach Brown et al. (2016)

\section{Ausgangssituation für das Recycling}

Die weltweite Bergwerksproduktion an Wolfram lag 2015 bei $80.900 t$ (Metallgehalt), wobei China mit einer Abbaumenge von 65.369t mit Abstand die wichtigste Rolle am Wolframmarkt zukommt. Die EU 36 förderten 2014 2,9\% der globalen Mengen, wobei $862 \mathrm{t}$ auf Österreich entfielen, dies entsprach einem Anteil von $33,6 \%$ des europäischen Abbaus. Die anschließende Abb. 2 verdeutlicht Österreichs Stellung in Hinblick auf die W-Gewinnung in Europa. Eine der bedeutendsten euro- päischen Wolframminen befindet sich in Mittersill (Brown et al. 2016, 2017; Bock 2017).

Die einzigartigen Eigenschaften von Wolfram, wie beispielsweise der hohe Schmelzpunkt $\left(3422^{\circ} \mathrm{C}\right)$, die außergewöhnlich hohe Zugfestigkeit bei Temperaturen über $1650^{\circ} \mathrm{C}$ und der geringe Ausdehnungskoeffizient führen dazu, dass dieses Metall in einer Vielzahl industrieller Anwendungen unerlässlich ist. Im Zuge verschiedenster thermischer und nasschemischer Prozesse, wie beispielsweise Oxidation, Laugung und Kristallisation erfolgt die Verarbeitung von Ausgangsmateriali- en wie Wolframerzen und Schrotten $\mathrm{zu}$ Ammoniumparawolframat (APW), dem wichtigsten Zwischenprodukt der Wolframindustrie. Die folgende Abb. 3 verdeutlicht die Komplexität der APWHerstellung anhand eines vereinfachten Flussdiagramms nach Lassner und Schubert (1999). Durch den hohen Schmelzpunkt von Wolfram kommen vor allem hydrometallurgische Verfahren zum Einsatz (British Geological Survey 2011; Lassner und Schubert 1999; Liedtke und Schmidt 2014).

Die Weiterverarbeitung von APW zu verschiedenen W-Oxiden erfolgt durch Kalzinierung an Luft (Wolframtrioxid, $\mathrm{WO}_{3}$ ) oder unter Luftausschluss-Bedingungen (Blauoxid, $\mathrm{WO}_{3-\mathrm{x}}$ ). Durch Reduktion der sehr reinen Oxide, welche vorwiegend unter Wasserstoffatmosphäre bei erhöhten Temperaturen stattfindet, lässt sich metallisches Wolfram erzeugen. Die bedeutendsten Endanwendungen von Wolfram stellen Hartmetallwerkzeuge, Metallprodukte, Legierungen, Chemikalien und Stahl dar. Die Studie von Leal-Ayala et al. aus 2015 fasst, wie Abb. 4 zu entnehmen ist, die Massenflüsse W-haltiger Stoffe von den Einsatzmaterialien bis zu den Enderzeugnissen im Jahr 2010 in 


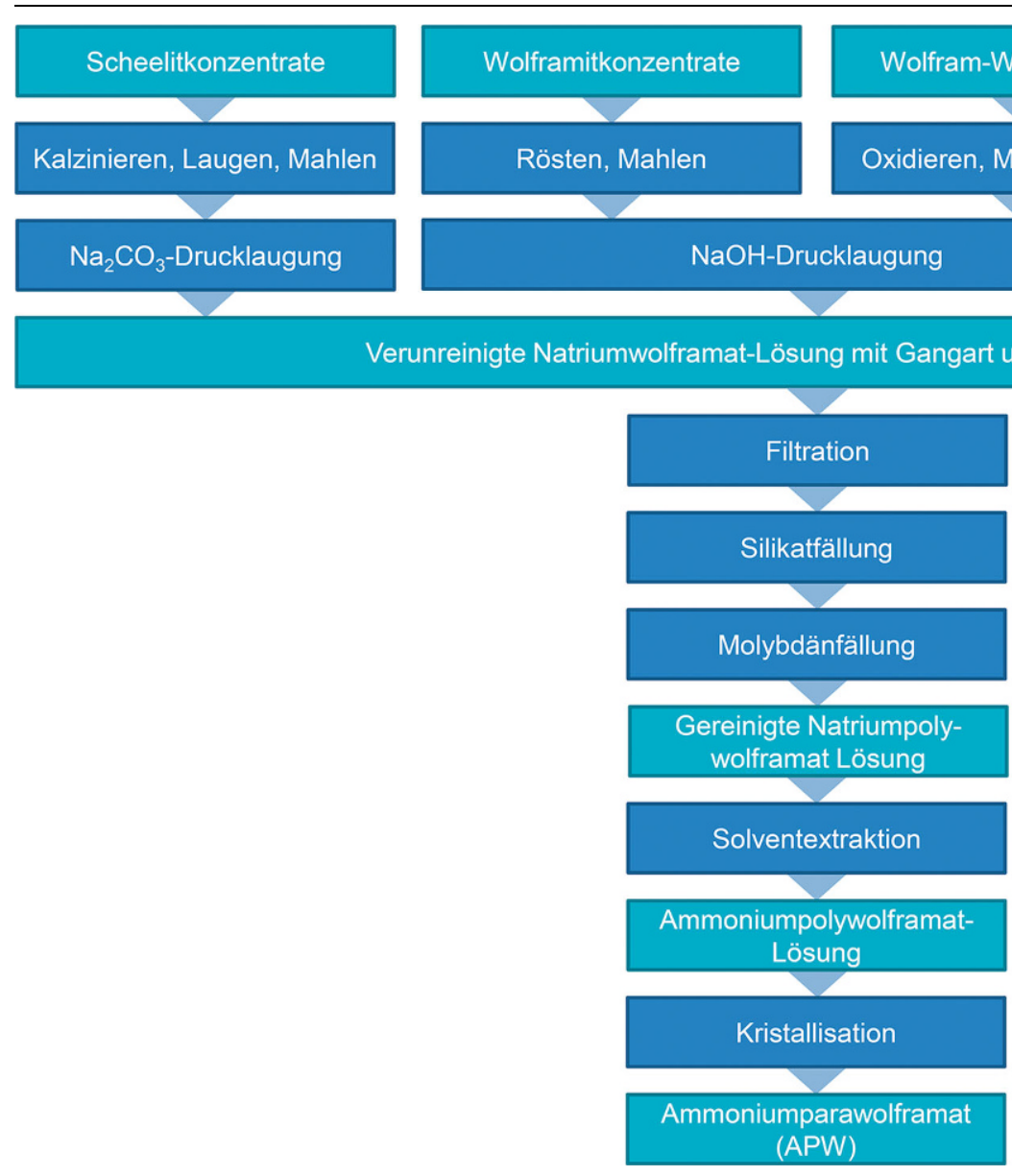

Abb. 3 Vereinfachtes Schema der APW-Herstellung aus W-haltigen Erzen und Schrotten nach Lassner und Schubert (1999)

einem Sankey-Diagramm zusammen und weist darüber hinaus Energieverbräuche sowie Wolframgehalte der einzelnen Produktsparten aus. Die Gruppe der Hartmetalle (HM) bzw. WC-Produkte stellt mit einem Anteil von 54\% das wichtigste Anwendungsgebiet für Wolfram dar (British Geological Survey 2011; Lassner und Schubert 1999; Liedtke und Schmidt 2014; Weil und Schubert 2013; Leal-Ayala et al. 2015).

Hartmetalle sind Verbundwerkstoffe, welche sich aus mindestens einer hochschmelzenden, harten, keramischen Phase sowie einem metallischen Binder, vorwiegend aus der Eisengruppe $(\mathrm{Co}, \mathrm{Ni}, \mathrm{Fe}$ ) zusammensetzen und pulvermetallurgisch produziert werden. Wolframkarbid (WC) stellt in den meisten Fällen die Hauptkomponente der Hartstoffphase dar. Die Herstellung von WC erfolgt über die Reaktion von Wolframpulver mit Kohlenstoff (reinster Ruß bzw. Grafit) unter reduzierender Wasserstoffatmosphäre im Zuge der Karburierung. Die Relevanz von Wolframkarbid für die österreichische
Wirtschaft lässt sich durch die Importbzw. Exportzahlen der Deutschen Rohstoffagentur (DERA) für dieses Material aus dem Jahr 2011 in Abb. 5 unterstreichen. Demnach führte Österreich mit ca. 1240 t 7,5\% der weltweiten Mengen von $16.451 \mathrm{t}$ ein. Hinsichtlich der ausgeführten Wolframkarbide (14.819t) stellt Österreich mit 5201 t und einem Anteil von $35,1 \%$ den größten Exporteur dar und übertrifft in diesem Bereich sogar China (23,2\%). Die Hauptabnehmer des österreichischen Wolframkarbids sind in Bezug auf die länderspezifischen Importdaten Großbritannien, Schweden und Deutschland mit jeweils 39, 28 bzw. $22 \%$ (Liedtke und Schmidt 2014; EMUGE-FRANKEN 2004; Böhlke 2002; Bose 2011; Prakash 2014; Wolfe et al. 2014; Schedler 1988)

Durch die Kombination der Härte des Wolframkarbids und der Duktilität des eingesetzten Bindemetalls sind Hartmetalle aufgrund ihrer herausragenden Eigenschaften, wie hohe Verschleiß- und Druckfestigkeit sowie gute Festigkeitswerte auch bei hohen
Temperaturen, zu einem unerlässlichen Werkzeug in der produzierenden Industrie geworden. Die Anwendungsgebiete für diesen Verbundwerkstoff erstrecken sich unter anderem von der Zerspanung metallischer und nichtmetallischer Materialien mit HM-Schneideinsätzen wie Wendeschneidplatten (WSP), über spanlose Umformung, Verschleißteile und Düsen bis hin zum Einsatz in der Tiefbohrtechnik, z.B. in Bohrkronen. Industriezweige wie Maschinenbau, metallverarbeitende Sektoren, Bau und Bergbau stellen die Hauptabnehmer für hartmetallbasierte Produkte dar (Lassner und Schubert 1999; Liedtke und Schmidt 2014; Prakash 2014; Schedler 1988; Schubert et al. 2010).

Das Recycling dieser Materialien am Ende ihres Produktlebenszyklus schafft einen wertvollen Beitrag zur Rohstoffsicherung und Ressourceneffizienz des hinsichtlich der Versorgung und wirtschaftlichen Bedeutung kritischen Rohstoffes Wolfram. Die Aufarbeitung von Hartmetallen bildet neben der Rückgewinnung von Edelmetallen aus sekun- 


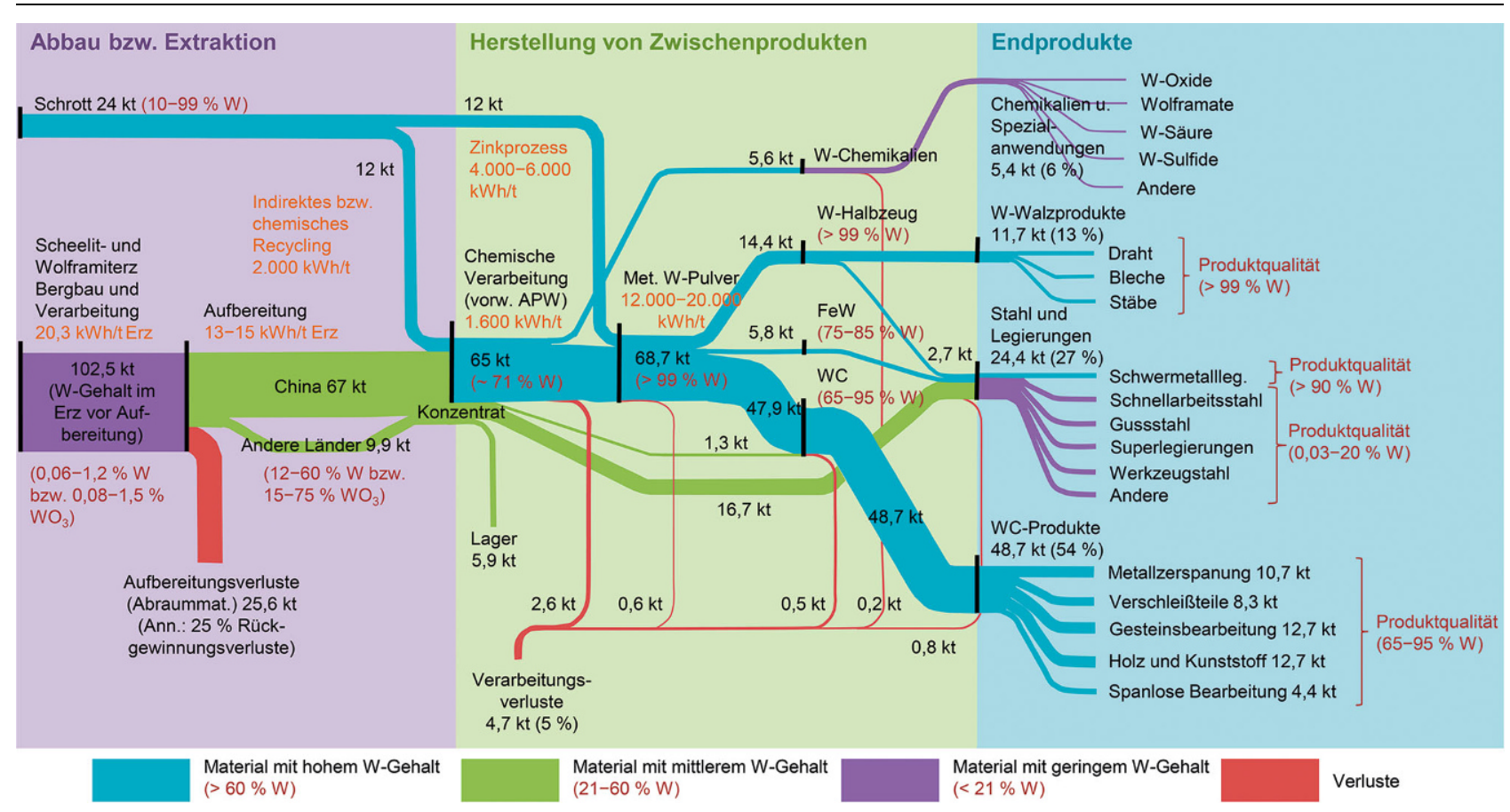

Abb. 4 Stoffflüsse wolframhaltiger Materialien im Jahr 2010 nach Leal-Ayala et al. (2015)

dären Quellen eine Ausnahme hinsichtlich industriell umgesetzter Recyclingtechnologien für kritische Materialen, welche ansonsten nur in speziellen Sparten bzw. für entsprechende Produktionsrückstände Anwendung finden (Luidold et al. 2013).

Neben strategischen Vorteilen resultiert daraus eine Vielzahl ökologischer und ökonomischer Chancen, welche sich aus der Rückgewinnung von W-haltigen Rezyklaten aus Hartmetallen ergeben, wie beispielsweise ein geringerer Energiebedarf durch den Einsatz von Sekundärmaterialien oder eine verbesserte Prozessausbeute. Im Bereich der Aufarbeitung von Hartmetallschrotten werden derzeit nach Gille und Meier (2012) je nach Region Recyclingraten von 50 bis $70 \%$ erzielt, wobei dieser Wert in der Sparte Wendeschneidplatten bei $90 \%$ liegt. Im Zuge der Aufarbeitung von Hartmetallen ist grundsätzlich zwischen direktem, semidirektem und indirektem Recycling zu unterscheiden, welche in Kap. 4 eingehender beschrieben werden (Luidold et al. 2013; Schubert et al. 2010; Oakes 1999; Gille und Meier 2012).

Die anschließende Abb. 6 verdeutlicht die Anteile unterschiedlicher Länder an den weltweiten Im- und Exporten von wolframhaltigen Abfällen und Schrotten im Jahr 2011 nach Liedtke und Schmidt (2014). Hinsichtlich der Importmengen befindet sich Österreich mit einem Anteil von $11 \%$ bzw. $1384 \mathrm{t}$ unter den drei größten Importeuren nach Deutschland und Großbritannien mit jeweils 45,8 bzw. $13 \%$. Ungefähr $70 \%$ der in Österreich eingeführten Mengen stammen aus dem Nachbarland Deutschland. Die wichtigsten Exporteure von Wolframabfällen und -schrotten sind die USA, Deutschland und Großbritannien (Liedtke und Schmidt 2014).

Die hohen Importdaten an W-haltigen Reststoffen verdeutlichen die Relevanz des Wolframrecyclings in Österreich. Das CD-Labor für Extraktive Metallurgie von Technologiemetallen unter der Leitung von Priv.-Doz. DI Dr. Stefan Luidold setzt sich daher am Lehrstuhl für Nichteisenmetallurgie der Montanuniversität Leoben intensiv mit Forschungs- und Entwicklungsarbeiten im Bereich des Recyclings von WCVerbundwerkstoffen auseinander. Der Fokus der laufenden Studien liegt vor allem auf grundlegenden Untersuchungen zu direkten Aufarbeitungstechnologien sowie semidirekten Verfahren zum Recycling von HM-Wendeschneidplatten und Bohrkronen aus der Öl- und Gasgewinnung, weshalb in Kap. 3 kurz auf deren Aufbau eingegangen wird.

\section{Aufbau ausgewählter WC-Verbundmaterialien}

Prinzipiell ist es möglich WC-Verbundmaterialien hinsichtlich ihres Aufbaus in die beiden Anwendungsgruppen Zerspanung metallischer Werkstoffe, wie beispielsweise Wendeschneidplatten, sowie Bearbeitung nichtmetallischer Stoffe (Öl- und Gasbohrungen usw.), wie z.B. Bohrkronen, zu unterteilen (Gee et al. 2014).

\subsection{Hartmetalle für die spanende Bearbeitung von Metallen}

Für die Zerspanung finden vor allem WC-Verbunde mit Cobalt als Binder Einsatz. Die Korngröße (KG) des Wolframkarbids kann sich hierbei von 0,2 bis $10 \mu \mathrm{m}$ erstrecken. Im Bereich der Binderphase sind Gehalte von 3 bis $25 \%$ Co möglich, gängige Sorten weisen jedoch 4 bis $16 \%$ metallischen Binder auf. Die anschließende Abb. 7 stellt Gefüge von Hartmetallen mittlerer Korngröße von 2,4 bis 2,5 $\mu \mathrm{m}$ mit einem Co-Gehalt von 12 bzw. $20 \%$ gegenüber (Prakash 2014; Fang et al. 2014; Ceratizit Austria S. A. 2015, 2016a, 2016b).

Die Eigenschaften der Hartmetalle werden stark durch ihren Aufbau hinsichtlich Korngröße und Bindergehalt bestimmt, wobei Härte, Festigkeit und Zähigkeit die wichtigsten Charakteristi- 

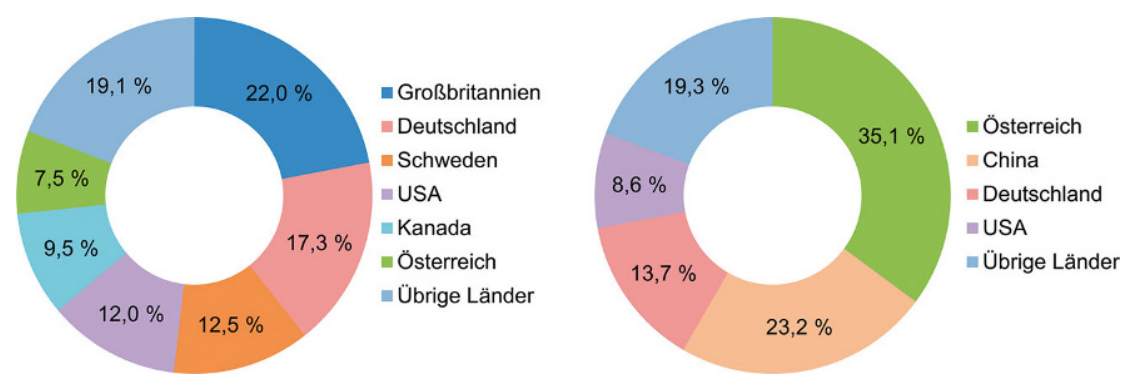

Abb. 5 Weltweite Importe (links) und Exporte (rechts) von WC im Jahr 2011 nach Liedtke und Schmidt (2014)
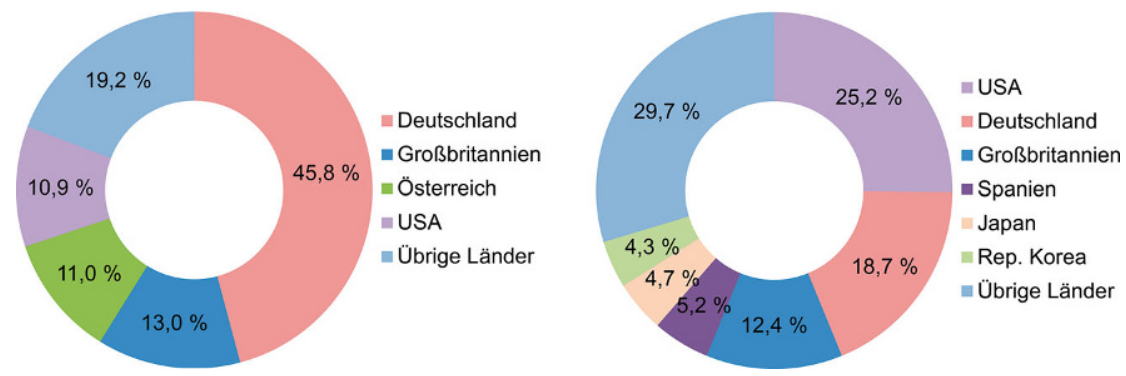

Abb. 6 Weltweitelmporte(links) und Exporte(rechts) von wolframhaltigenAbfällen und Schrotten im Jahr 2011 nach Liedtke und Schmidt (2014)
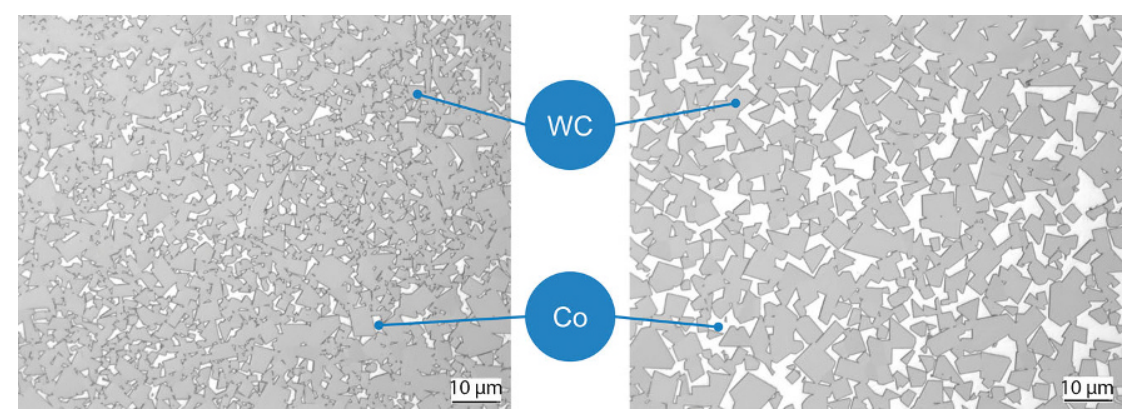

Abb. 7 Gefüge von Hartmetallen mit einer mittleren Wolframkarbidkorngröße von 2,4 bis 2,5 $\mu$ mund einem Cobaltgehaltvon 12 \% (links) bzw. $20 \%$ (rechts) nachEbner(2016)

ka darstellen. Beispielsweise zeichnen sich Verbunde mit geringem Anteil an Co und gleichzeitig kleiner KG durch sehr hohe Härtewerte aus (Prakash 2014; Ceratizit S. A. 2015).

Die Herstellung von Hartmetallen erfolgt nach einem pulvermetallurgischen Prozess, in welchem Wolframkarbid mit dem Bindermetall und nach Bedarf mit weiteren Komponenten, wie z. B. TiC, TaC usw., in der gewünschten Zusammensetzung gemischt und gemahlen werden. Daraufhin folgt ein Pressen des resultierenden Pulvers in die jeweilige Form und ein Sintern der Grünlinge zu sogenannten gesinterten Karbiden. Aufgrund der hohen thermischen und abrasiven Belastungen, welche bei spanender Bearbeitung von Metallen auftreten, sind Schneidein- erstmals 1973 von General Electric Co. erzeugt wurden, erfreuen sich stark steigender Bedeutung (Scott 2015; Adams und Charrier 1985).

Die Bohrmeißel bestehen aus einem Verbindungsstück aus Stahl, auf welchem das Gewinde für den Bohrstrang aufgebracht ist, aus dem eigentlichen Kopfteil, welcher aus Hartmetall besteht, sowie den Schneideinsätzen aus polykristallinem Diamant-Komposit (PDC) (siehe Abb. 8). Der hartmetallhaltige Kopf unterteilt sich in einen leicht zerspanbaren Schulterteil aus infiltriertem Wolfram sowie dem Kopfund Seitenteil aus extrem hartem infiltriertem Wolframkarbid und -schmelzkarbid (CTC) (DIAPAC 2012; Oerlikon Metco 2014).

Die zylindrischen PDC-Schneidaufsätze tragen das Gestein durch Scheren ab, sodass der Hartmetallkopf hauptsächlich über Erosion durch das Bohrfluid sowie Abrasion durch losgelöstes Gestein und zugesetzte Reibpartikel beansprucht wird (Azar und Robello Samuel 2007; Adams und Charrier 1985). Um eine hohe Biegebruch- und Verschleißfestigkeit mit guter Erosionsbeständigkeit $\mathrm{zu}$ verbinden, werden grobe, mit einer Kupferlegierung infiltrierte Wolfram-, Wolframkarbid- und Wolframschmelzkarbidpulver verwendet (Kembaiyan und Oldham 2001a; Oerlikon Metco 2014).

Das wolframhaltige Pulver wird in eine Gussform aus Grafit um einen Stahlrohling herum geschichtet und durch Vibration verdichtet. Gemeinsam mit den über der kompakten Schüttung bereitgestellten Bindermetallwürfeln wird die Mischung auf die Infiltrationstemperatur erhitzt, sodass sich der flüssige Binder an das Pulver anlegt. Nach dem kontrollierten Abkühlvorgang bildet sich ein fester, porenfreier Werkstoffverbund aus durch die Kupferlegierung gebundenen Wolfram-, Wolframkarbid- und Wolframschmelzkarbidkörnern (siehe Abb. 9) (De Maindreville und Gomez 2015; Kembaiyan und Oldham 2001b; Uno et al. 2011).

Wolframschmelzkarbid bildet einen wesentlichen Bestandteil in PDC-Bohrköpfen, da es besonders hart und verschleißfest ist. Es stellt eine eutektische Mischung aus Monowolframkarbid (WC) und Diwolframkarbid $\left(\mathrm{W}_{2} \mathrm{C}\right)$ dar und entsteht durch das schockartige Erstarren einer Wolfram-Kohlenstoff-Schmelze, bei der $\mathrm{W}_{2} \mathrm{C}$-Kristalle in Form feinster Lamellen ausgeschieden werden. Das Material wird anschlie- 


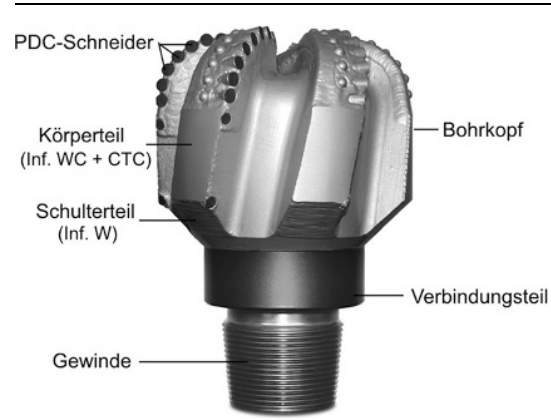

Abb. 8 Aufbau eines PDC-Bohrkopfs nach VAREL Oil \& Gas Drill Bits (2017)

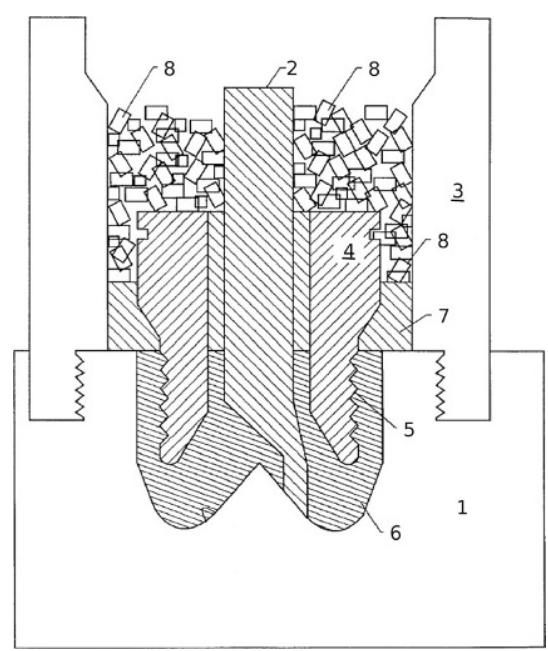

Abb. 9 Herstellung eines PDC-Bohrkopfs. 1 Grafitform, 2 Sandkern für Bohrfluidgang, 3 Stahltrichter, 4 Stahlrohling mit Einkerbungen 5, 6 WC/CTC-Pulver, 7W-Pulver, 8Bindelegierungswürfel nach Kembaiyan und Oldham (2001b)

ßend in unregelmäßige Körner gebrochen (Kurlov und Gusev 2013; Starck 2015).

Die Schliffbilder in Abb. 10 und 11 verdeutlichen, dass sich die verschiedenen Wolframphasen, aus denen ein Bohrmeißel besteht, stark in ihrer Form, Struktur sowie Korngröße differenzieren. Aus diesem Grund erhöht sich die Komplexität der Anforderungen an den Recyclingprozess, bei dem die Rückführung der einzelnen Komponenten in den Wertstoffkreislauf gewährleistet werden muss.

\section{Recyclingvarianten}

Der Großteil der anfallenden Schrotte weist höhere Wolframkonzentrationen als typische Erze, wie beispielsweise Wolframit oder Scheelit auf, deren durchschnittlicher Gehalt bei $<1 \% \mathrm{WO}_{3}$ liegt. Daraus resultieren vor allem wirtschaftliche Anreize, wodurch besonders Produktionsrückstände in den Fokus von Recyclingbestrebungen rücken. WC-Verbund-Schrotte fallen vor allem im Bereich der Vorstoffproduzenten, bei der HM-Herstellung, im Werkzeugbau oder im Zuge der Anwendung an. In Abhängigkeit von der Konsistenz lässt sich bei hartmetallhaltigem Sekundärmaterial zwischen Weich- und Hartschrott unterscheiden. Materialien der ersten Gruppe entstehen in großen Mengen vor allem im Rahmen der Hartmetallproduktion und umfassen pulverförmige Stoffe, wie z. B. Schleifschlämme, Kehrabfälle oder Grünlinge. Durch das Anfallen dieser Rückstände im eigenen Herstellungsprozess ist es möglich, einen Teil der Materialströme sauber und sortenrein zu halten, weshalb eine effiziente Rückführung in den Wertstoffkreislauf erfolgen kann (Luidold et al. 2013; Zeiler 1997).

Die Fraktion der Hartschrotte enthält gesinterte Hartmetalle, wie Verschleiß- oder fehlgesinterte Teile sowie Schneid- und Umformwerkzeuge am Ende ihres Produktlebenszyklus. WC-Verbundwerkstoffe weisen einen Wolframgehalt im Bereich von 70 bis $97 \%$ auf und sind sowohl auf ökologischer als auch ökonomischer Basis zweckmäßig rezyklierbar. Im Gegensatz zu den Weichschrotten fallen diese Materialien hauptsächlich im Anwendungsbereich an, zudem sind aufgrund verschiedenster Anforderungen an das Werkzeug weitgehend unterschiedliche HM-Sorten im Einsatz. Eine große Herausforderung besteht daher in der Sortenreinheit der Hartschrottfraktion, da für ein industrielles Recycling entsprechende Mindestmengen erforderlich sind, welche sich oftmals nur durch Sammlung mehrerer Anwender erzielen lässt. Der äußere Stoffkreislauf wird durch die Gruppe der Hartschrotte dominiert und bestimmt somit das eigentliche Recycling (Gille und Meier 2012; Zeiler 1997; Gille et al. 2012).

Für die Aufarbeitung von WC-Verbundwerkstoffen lässt sich grob zwischen Down- und Recycling unterscheiden, wobei ersteres die Herstellung von Ferrowolfram sowie von Beschichtungen umfasst. Für das industrielle Recycling stehen verschiedenste Verfahrensvarianten zur Verfügung, welche zumeist auf einer Kombination mechanischer, physikalischer, chemischer und elektrochemischer Methoden basieren. Das Recycling gliedert sich wiederum in indirekte, direkte und semidirekte Verfahren (Gille und Meier 2012; Gille et al. 2012; Angerer et al. 2011a).

Indirekte bzw. chemisch-hydrometallurgische Technologien zielen darauf ab, die sekundären Rohstoffe auf ihr atomares Niveau aufzuschließen, woraus elementare Metalle oder Metallpulver resultieren. Dazu folgt nach einer Oxidation der Materialien die gesamte hydrometallurgische Produktionsroute, welche Abb. 3 zu entnehmen ist (Gille et al. 2012; Gille und Meier 2012; Angerer et al. 2011a).

Im Zuge direkter Recyclingmethoden erfolgt eine physikalisch-mechanische Zerlegung der WC-Verbunde, wodurch sowohl das Karbid als auch der Binder intakt gehalten werden. Typische Beispiele für diese Route stellen der Zinkund der Coldstreamprozess dar (Angerer et al. 2011a; Gille und Meier 2012; Gille et al. 2012; Lassner und Schubert 1999).

Semidirekte Varianten umfassen die Überführung einer Komponente in ein wässriges Medium, wohingegen die andere nach dem Prozess nach wie vor fest vorliegt. Das selektive Lösen des Binders lässt sich z. B. durch das Laugen mittels unterschiedlichen Säuren oder über elektrolytische Verfahren im sauren Medium realisieren. Das ungelöste WC kann wieder in der Erzeugung von Hartmetallen eingesetzt werden (Lassner und Schubert 1999).

Die anschließenden Kapitel gehen näher auf die einzelnen Varianten zum Recycling von WC-Verbundmaterialien ein.

\subsection{Indirektes Recycling}

Im Rahmen des indirekten oder chemisch-hydrometallurgischen Recyclings werden unter anderem Schleifschlämme, Kehrichte, feste Materialien usw. behandelt, welche aufgrund ihres hohen Verunreinigungsgrades mit anderen Verfahren ökonomisch nicht sinnvoll rezyklierbar sind (Lassner und Schubert 1999).

Grundsätzlich erfolgt als erster Schritt eine Oxidation der Rückstände an Luft, mit Chemikalien oder elektrischer Energie. Dies überführt das Wolfram in eine im alkalischen Medium lösliche hexavalente Konfiguration, welche den Ausgangspunkt für die anschließende hydrometallurgische Aufarbeitungsroute bildet. Der weiterführende Prozess gleicht jener der APW-Herstellung, dem wichtigsten 


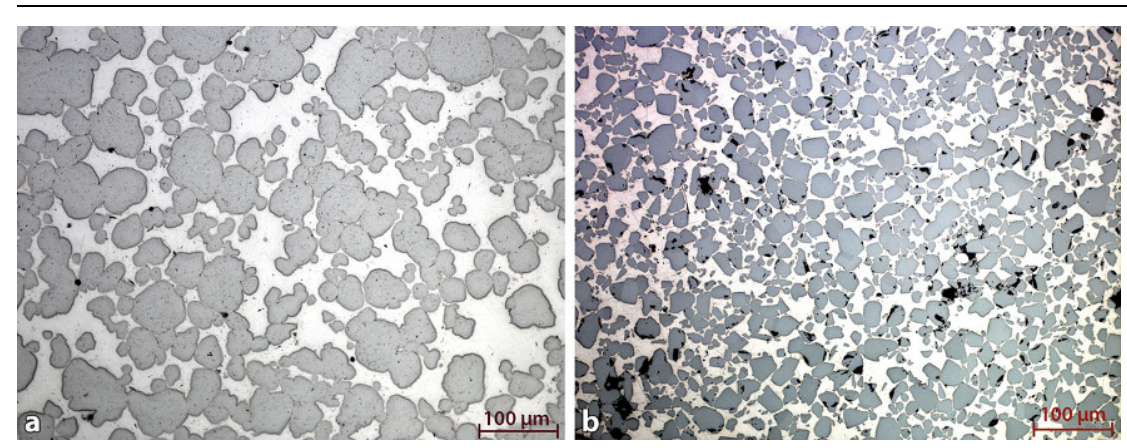

Abb. 10 Lichtmikroskopaufnahmen von infiltriertem Wolfram(a) und infiltriertem Wolframkarbid (b)

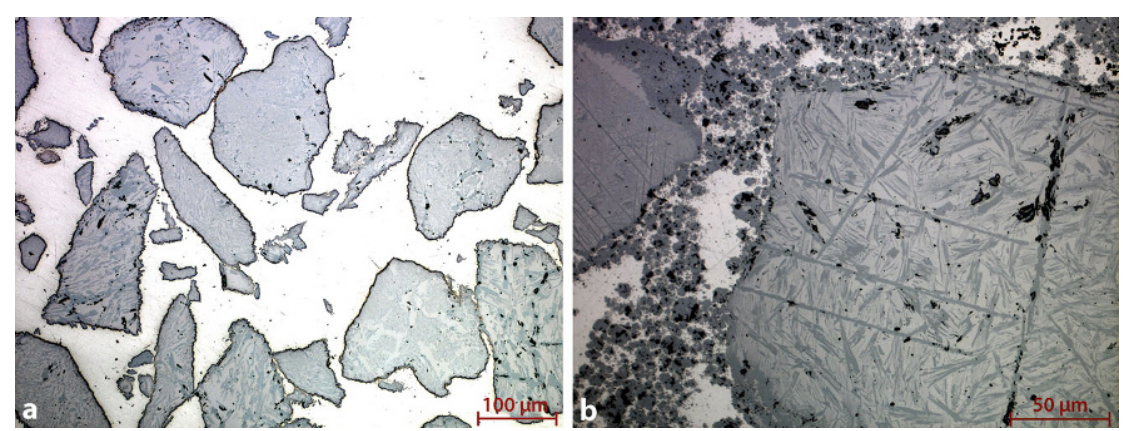

Abb. 11 Lichtmikroskopaufnahmen von infiltriertem Wolframschmelzkarbid (a) und einer infiltrierten Mischung aus Wolframschmelzkarbid und WC (b)
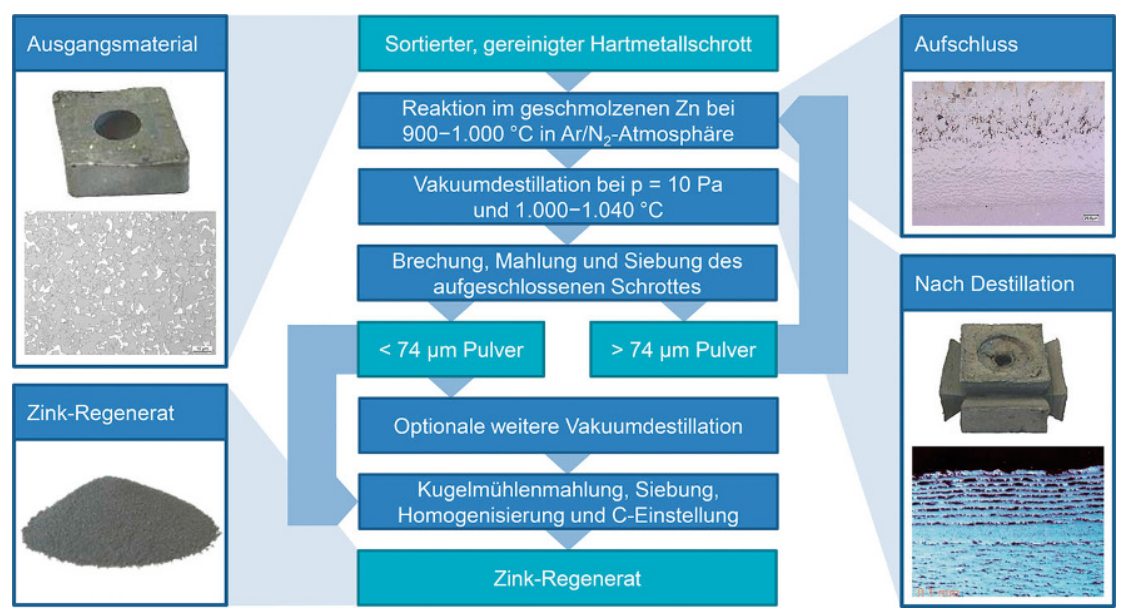

Abb. 12 Fließbild des Zinkprozesses nach Angerer et al. (2011a) mit Darstellung der entsprechenden Zwischenprodukte (Tikomet Oy 2017) und Schliffbilder (Ebner 2016; Ebner et al. 2017; Brookes 2014)

Zwischenprodukt der W-Produktion, welches bereits in Kap. 2, Abb. 3 beschrieben wurde (Lassner und Schubert 1999).

Diese Verfahrensvariante bietet aufgrund der Vielzahl an hydrometallurgischen Prozessstufen die Möglichkeit, Metalle, wie beispielsweise Cobalt, Nickel oder Silber, zu entfernen. Daher liefert das indirekte Recycling trotz des an Chemikalien, welche den Vorteilen der hohen Produktreinheit gegenüberstehen (Lassner und Schubert 1999; Gille und Meier 2012).

Typische Verfahren für diese Recyclingsparte stellen unter anderem der alkalische Schmelzaufschluss mit $\mathrm{Na}$ Salzschmelzen, das oxidierende Rösten mit anschließender alkalischer Laugung oder auch der Axel-Johnson-Prozess dar (Angerer et al. 2011b).

\subsection{Direktes Recycling}

Direkte Verfahren zum Recycling von WC-Verbundmaterialen sind dadurch gekennzeichnet, dass das Wolframkarbid sowie der Binder intakt gehalten und somit in Pulver zerlegt werden, deren Zusammensetzung jener des Ausgangsstoffes entspricht. Das heißt, dass kein chemischer Aufschluss, sondern eine physikalisch-mechanische Behandlung der Schrotte erfolgt. Klassische Beispiele für diese Variante stellen der Zinkprozess, das ColdstreamVerfahren oder auch die Bloating/ Crushing-Methode bzw. deren Kombinationen dar (Lassner und Schubert 1999; Angerer et al. 2011a).

Als wichtigste indirekte Recyclingvariante ist aufgrund der sehr hohen W-Ausbeute, der Umweltfreundlichkeit und den entsprechenden Mengen von $12.000 \mathrm{t}$ im Jahr 2010 der Zinkprozess anzuführen. Dieser wurde in den 1970er-Jahren entwickelt (Barnard et al. 1969) und findet vor allem seit 2005 immer breitere Beachtung, wodurch heutzutage $10 \%$ der in Europa genutzten WC-Co-Pulver ein Rezyklat aus diesem Verfahren darstellen, wohingegen dieser Wert 2005 bei nur $5 \%$ lag. Als Ausgangsmaterialien finden vor allem Schneideinsätze, jedoch seit 2005 unter anderem auch Stäbe, Bohrer und Verschleißteile Einsatz (Leal-Ayala et al. 2015; Angerer et al. 2011a; Karhumaa und Kurkela 2013).

Im Zinkprozess werden Hartmetalle im ersten Schritt mit flüssigem Zink oder Zn-Dampf bei 800 bis $1050^{\circ} \mathrm{C}$ unter Stickstoff- oder Argonatmosphäre mit einem typischen HM:Zn-Verhältnis von 1:1,3 in Grafittiegeln aufgeschlossen. Das flüssige Zink infiltriert das WCCo-Material und bildet intermetallische Phasen mit dem Binder aus. Diese Verbindungen zeigen im Vergleich zu Cobalt eine höhere Volumsausdehnung, wodurch der ursprüngliche Verbund aufgebläht und zerstört wird und als Produkt ein poröser Schwamm vorliegt. 

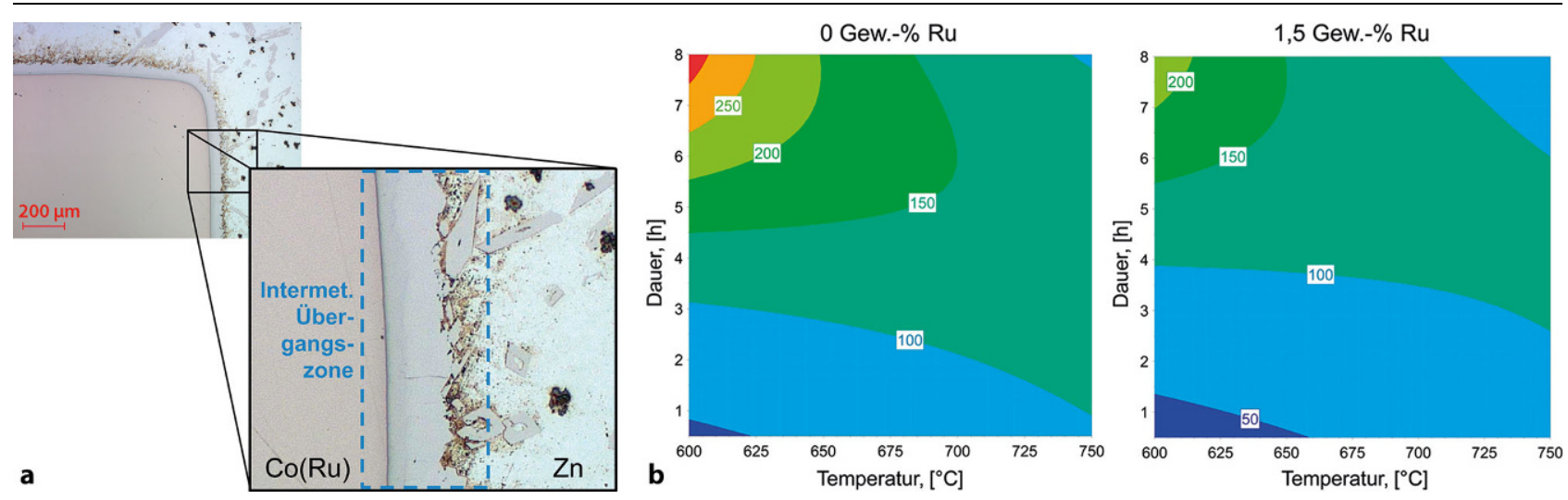

Abb. 13 Aufnahme der intermetallischen PhasenzwischenCo(Ru) und Zink (a) sowie Darstellung der Gesamtschichtdicke derÜbergangszone in $\mu \mathrm{m}$ in Abhängigkeit von Temperatur und Dauer bei 0 (b, Mitte) und 1,5 Gew.-\% Ru (b, rechts) (Ebner et al. 2017)

Die typische Infiltrationsgeschwindigkeit der Zinkschmelze liegt bei $1 \mathrm{~mm} / \mathrm{h}$, mit Zn-Dampf lassen sich wesentlich höhere Werte erzielen (Angerer et al. 2011a; Gille und Meier 2012; Karhumaa und Kurkela 2013; Lassner und Schubert 1999; Alkatsev et al. 2008).

Der zweite Schritt dieses Verfahrens sieht eine Abtrennung des Zinks durch Destillation auf Basis einer Temperaturerhöhung auf 1000 bis $1050^{\circ} \mathrm{C}$ und das Anlegen eines Vakuums im Bereich von 10 Pa vor. Das verdampfende Zink lässt sich durch Kondensation zurückgewinnen und somit wiederverwenden. Der Rückstand weist eine poröse, blättrige Struktur auf, wodurch eine Aufbereitung des Materials durch Brechen, Mahlen und Sieben möglich wird. Das entstehende Pulvergemisch kann zur Herstellung von Hartmetallen durch Pressen und Sintern herangezogen werden (Karhumaa und Kurkela 2013; Lassner und Schubert 1999; Gille und Meier 2012; Angerer et al. 2011a).

Die Abb. 12 stellt den Ablauf des Zinkprozesses anhand eines Fließbilds dar und verdeutlicht die einzelnen Schritte durch Ausgangs-, Zwischenund Endprodukte sowie entsprechende Schliffbilder.

Während des Zinkprozesses ist es nicht möglich, Verunreinigungen, welche mit dem Ausgangsmaterial oder im Zuge des Verfahrens eingebracht werden, zu entfernen, da kein Raffinationsschritt vorhanden ist. Dadurch reichern sich diese Kontaminationen bei mehrmaligem Durchlaufen des Recyclingprozesses an (Gille und Meier 2012)

\subsection{Semidirektes Recycling}

Das Verfahrenscharakteristikum besteht in der Binderlösung, wobei die Hartphase (WC) in Form eines Karbidskeletts übrig bleibt. Ohne den Binder lässt sich das spröde WC vergleichsweise einfach pulverisieren und dann erneut zur Hartmetallproduktion einsetzen. Um dies zu bewerkstelligen, gibt es einerseits chemische, chemischmechanische sowie elektrochemische Methoden und im Speziellen den Menstruum-Prozess. Abgesehen vom Menstruum-Verfahren spielen derzeit die semidirekten Techniken keine großtechnische Rolle. Allerdings offerieren diese Vorzüge, wie minimierte Prozesssowie Investitionskosten, geringeren Energiebedarf und niedrigere Umweltbelastung. Besonders in Gegenüberstellung mit der aufwendigen indirekten Recyclingroute lassen sich zahlreiche Verfahrensschritte vermeiden (Gille und Meier 2012; Schiesser 2003; Meyer 1979; Shwayder 1969; Edtmaier et al. 2005; Ritsko et al. 1982; Gürmen et al. 2005).

Die meisten Informationen über diese Gruppe liefern Patente, aber es gibt auch vereinzelt wissenschaftliche Berichte. Wegen des beschränkten Wissensstandes können nur wenige allgemeingültige Aussagen getroffen werden. Die ersten beiden Konzepte verwenden zumeist wässrige Säuren als Basis, etwa $\mathrm{HNO}_{3}$ (Gürmen et al. 2005), $\mathrm{HCl}$ (Kojima et al. 2005), $\mathrm{H}_{3} \mathrm{PO}_{4}$ (Shwayder 1969), $\mathrm{H}_{2} \mathrm{SO}_{4}$ (Kojima et al. 2005) bzw. Essigsäure (Edtmaier et al. 2005) aber auch $\mathrm{NH}_{4} \mathrm{OH}$ (Shwayder 1972).

Vorteilhaft auf die Reaktionsgeschwindigkeit soll eine mechanische Einwirkung sein, welche das Karbidskelett abträgt und somit die Diffusi- onsbarriere verkleinert. Zur Beschleunigung verweisen die Publikationen zumeist auch auf einen Vorbehandlungsschritt (Zerkleinerung) bzw. finden Grünschrotte Einsatz. Positiv wirkt sich auch eine Temperaturerhöhung aus, teilweise eine Konzentrationssteigerung des Laugungsmittels sowie das Einblasen von Sauerstoff, jedoch bestehen immer Wechselwirkungen mit der Lösung und der Hartmetallsorte, welche nicht genau bekannt sind. Eine Herausforderung stellt die Bildung von $\mathrm{WO}_{3} \cdot \mathrm{H}_{2} \mathrm{O}$ in sauren Medien dar. Neben der Kontaminierung des Rezyklats verkleinert dieses auch die freien Kanäle im Karbidskelett und setzt daher die Laugungsgeschwindigkeit herab (Gürmen et al. 2005; Kojima et al. 2005; Shwayder 1969; Edtmaier et al. 2005; MacInnis und Vanderpool 1976).

Bei den elektrochemischen Techniken kommen neben sauren Elektrolyten, wie beispielsweise $\mathrm{HNO}_{3}$ (Lin et al. 1996), $\mathrm{H}_{3} \mathrm{PO}_{4}$ (Ghandehari 1980), Essigsäure (Ghandehari 1980), $\mathrm{HCl}$ (Lin und Lee 1995) und $\mathrm{H}_{2} \mathrm{SO}_{4}$ (Paul et al. 1985), auch basische wie etwa ammoniakalische Lösungen (Vadasdy et al. 1986) sowie $\mathrm{NaOH}$ oder $\mathrm{KOH}$ (Vanderpool 1983) zum Einsatz. In diesem Fall oxidiert und löst sich das Bindermetall an der Anode und scheidet sich anschließend wieder an der Kathode ab. Jedoch geht auch immer etwas Wolfram in Lösung, welches ebenfalls kathodisch reduziert wird oder $\mathrm{zu} \mathrm{WO}_{3}$ bzw. $\mathrm{WO}_{3} \cdot \mathrm{H}_{2} \mathrm{O}$ reagiert, welches auf dem Karbidskelett verbleibt. Dadurch besteht auch das Risiko einer anodischen Passivierung. Diverse Zusätze wie $\mathrm{NH}_{4} \mathrm{Cl}$, $\left(\mathrm{NH}_{4}\right)_{2} \mathrm{SO}_{4}$, $\mathrm{NH}_{4} \mathrm{NO}_{3}, \mathrm{NaClO}_{4}$ sollen diese Gefahr unterbinden.

Das US-Patent 2529778 (McKenna 1950) illustriert die Grundlagen für den 


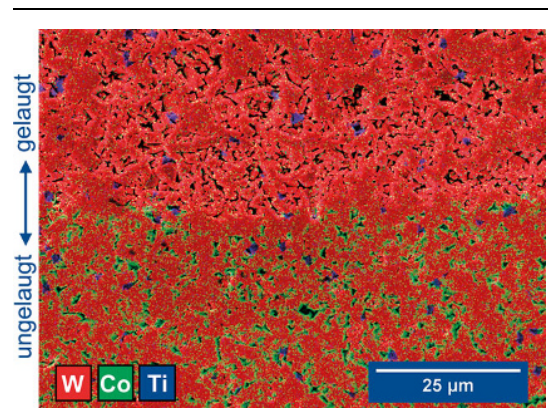

Abb. 14 Übergang zwischen binderfreier Zone und Ausgangsmaterial (Kücher und Luidold 2017)

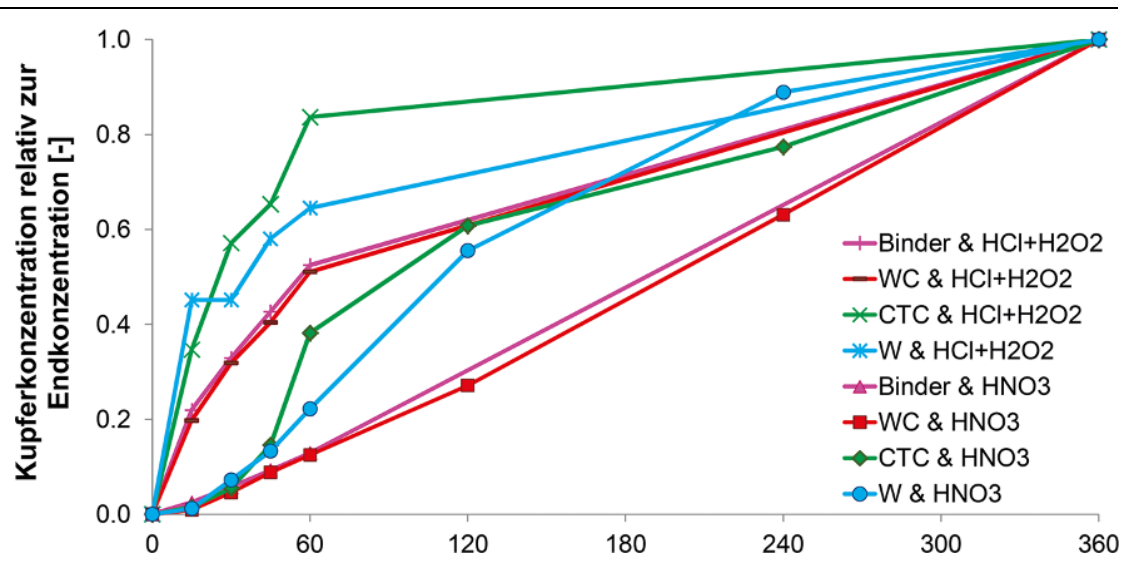

Abb. 15 Relative Kupferkonzentration unterschiedlicher Substrate in $\mathrm{HNO}_{3}$ und $\mathrm{HCl}$ mit $\mathrm{H}_{2} \mathrm{O}_{2}$

gen etc.) für unterschiedliche Sorten zunehmend an seine Grenzen. Aus diesen Gründen befasst sich das Christian Doppler Labor für Extraktive Metallurgie von Technologiemetallen intensiv mit den Grundlagen zu den einzelnen Prozessschritten mehrerer Verfahrensrouten, um ein vertieftes und detaillierteres Verständnis der dabei relevanten Vorgänge $\mathrm{zu}$ erhalten. Diese bilden letztendlich die Ausgangsbasis für den Fortschritt dieser Technologien.

\subsection{Phasenausbildung im Zinkprozess}

Im Zuge des Zinkprozesses bilden sich gemäß dem binären Co-Zn-System unterschiedlichste intermetallische Phasen zwischen dem festen Cobaltbinder und dem flüssigen Zink aus. Durch das höhere Volumen dieser Verbindungen im Vergleich zu Co erfolgt eine Aufblähung und Desintegration des Hartmetallverbundes in der ersten Stufe dieser Recyclingtechnologie. Da der exakte Mechanismus des Aufschlusses bisher nicht bekannt ist, sollen die Studien des Lehrstuhls für Nichteisenmetallurgie fundierte Informationen $\mathrm{zu}$ dieser Thematik liefern. Aktuelle Arbeiten (Ebner et al. 2017; Ebner 2016) beschäftigen sich vor allem mit den entstehenden Phasen zwischen den beiden Hauptkomponenten Cobalt und Zink. Die Vereinfachung des Systems, welches eine Behandlung des Bindermetalls mit einer Zinkschmelze vorsieht, ermöglicht grundlegende Einblicke in die Vorgänge und Abläufe im ersten Schritt des Zinkprozesses. Einen weiteren wichtigen Schwerpunkt stellt die Evaluierung des Einflusses von weiteren Binderlegierungselementen auf die Phasenausbildung im Co-
Zn-Diffusionspaar, wie beispielsweise Ruthenium, dar.

Im Zuge der Untersuchungen erfolgte die Kontaktierung von Cobalt mit bestimmten Ru-Gehalten von 0 bis 1,5 Gew.-\% mit flüssigem Zink bei bestimmten Temperaturen $(600$ bis $750^{\circ} \mathrm{C}$ ) für 0,5 bis $8 \mathrm{~h}$, woraus eine entsprechende Ausbildung von intermetallischen Phasen resultierte. Die Gesamtschichtdicke der entstehenden Verbindungen ließ sich mittels Lichtmikroskopie (LIMI) bestimmen. Die Analyse der generierten Daten wurden unter Anwendung der Statistiksoftware Modde 11 durchgeführt, welche eine übersichtliche Darstellung der Ergebnisse sowie eine eingehende Analyse der Auswirkung der einzelnen Einflussgrößen zulässt. Die Abb. 13 enthält ein Beispiel einer Übergangszone zwischen $\mathrm{Co}(\mathrm{Ru})$ und $\mathrm{Zn}$ und verdeutlicht die $\mathrm{Ab}$ hängigkeit der Gesamtschichtdicke der gebildeten Schichten von der Temperatur und der Dauer für 0 und 1,5 Gew.\% Ruthenium. Es wird deutlich, dass sich die breitesten Lagen bei niedrigen Temperaturen und gleichzeitig hoher Dauer erzielen ließen. Ein steigender Ru-Gehalt führt zu dünneren Schichten, woraus abzuleiten ist, dass Ru das Wachstum der Lagen tendenziell hemmt. Die Ergebnisse dieses vereinfachten Systems führen zur Annahme, dass die breitere Übergangszonen das Eindringen des Zinks im realen WCCo-Verbund im Zuge des Zn-Prozesses und somit die Desintegrationsrate des Hartmetalls verlangsamen. Durch einen geringeren Diffusionswiderstand beschleunigen dünnere Lagen die Auflösung des Materials durch die Penetration des Zinks, jedoch haben sie keine positive Auswirkung auf das Ab- 
platzen der lamellaren WC-Strukturen, wodurch die Zerstörung des Verbundes nicht vorangetrieben wird. Weitere Untersuchungen auf diesem Gebiet sollen Aufschluss über die ideale Schichtdicke geben, welche die Desintegration von Hartmetallen optimal vorantreibt.

Neben der Auswertung und Interpretation der Gesamtschichtdicke, welche ein Thema der Forschungstätigkeiten in diesem Bereich darstellt, wurden limitierende Faktoren anhand des physikalisch-chemischen Ansatzes identifiziert sowie der Einfluss des Rutheniums auf die Auflösung des Cobalts im flüssigem Zink eingehend evaluiert. Die Analyse der Resultate gab Aufschluss über Diffusionsbarrieren und die Auswirkung des zusätzlichen Binderlegierungselements $\mathrm{Ru}$ in Hinblick auf das Wachstum der intermetallischen Phasen im Co-ZnDiffusionspaar.

Weiterführende Arbeiten auf diesem Gebiet stellen die Evaluierung des Desintegrationsverhaltens von Hartmetallen im Zuge des Zinkprozesses in den Vordergrund. Diese Studien basieren auf den Erkenntnissen der grundlegenden Untersuchungen zur Phasenausbildung in der ersten Stufe des ZnVerfahrens und sollen Aufschluss über das Verhalten von WC-Co-Verbunden bei Behandlung mit flüssigem Zink unter besonderer Berücksichtigung unterschiedlicher Parameter geben. Diese Untersuchungen zielen darauf ab, fundierte Aussagen zur Auswirkung verschiedenster Einflussgrößen auf den Desintregrationsgrad von Hartmetallen während des Aufschlusses zu generieren. Die Resultate sollen einen wesentlichen Beitrag zur gezielten Prozessoptimierung liefern und so das direkte Recycling von WC-Verbunden nachhaltig verbessern und effizienter gestalten.

\subsection{Semidirektes Recycling von HM-WSP}

Wie bereits angeführt, beruhen die semidirekten Recyclingkonzepte auf dem selektiven Lösen des Binders mittels hydrometallurgischer Methoden. Entsprechend gilt es die Hartphase, das WC, möglichst unbeeinflusst zu hinterlassen um diese später in zerkleinerter Form wieder einsetzen zu können. Jedoch bestehen noch einige Hindernisse, welche die industrielle Umsetzung bislang nicht gestatteten. So existiert die Gefahr der partiellen Oxidation der Karbide (Schiesser 2003; Meyer 1979;
Shwayder 1969), der langwierigen Binderlösung (Schiesser 2003; Shwayder 1969) und von nicht entfernbaren Beschichtungen (Kopylov und Polyakov 1999), welche die Rezyklatqualität mindern. Trotzdem bietet dieser Ansatz den Vorteil, die aufwendige Primärroute zu umgehen. Daher sollen die nachfolgend vorgestellten Untersuchungen am CDL für Extraktive Metallurgie von Technologiemetallen dazu die Grundlagen schaffen.

Ausgehend von einer umfassenden Literatur- sowie Patentrecherche kamen Säuren, $\mathrm{H}_{2} \mathrm{O}_{2}$ sowie Additive in Tastversuchen (Kücher et al. 2016) zum Einsatz. In einem Zeitraum von $4 \mathrm{~h}$ bei $50^{\circ} \mathrm{C}$ wurden verschiedene Lösungen und Mischungen zur Binderlaugung eingesetzt. Hierzu zeigt Abb. 14 die Reaktionsfront zwischen gelaugtem und ungelaugtem Substrat. Zusammenfassend lässt sich festhalten, dass Säuren ohne Oxidationsmittel keine Wirkung auf das Substrat erzielten. Komplexere Zusammensetzungen erreichten einen mäßigen Abtrag mit einhergehender Gasentwicklung. Nur organische sowie anorganische Säuren in Verbindung mit $\mathrm{H}_{2} \mathrm{O}_{2}$ bewirkten teilweise einen Abtrag als auch eine bevorzugte Binderlösung.

Darauf aufbauend unterstützte das Statistikprogramm Modde 11 die Entwicklung eines Versuchsplans. Eine umfassendere Darstellung der Experimente zur Bewertung der Einflüsse von Säure, $\mathrm{H}_{2} \mathrm{O}_{2}$ und Temperatur auf das Ergebnis der Laugung findet sich bei Kücher et al. (2017a, 2017b ). Zur Beobachtung des Reaktionsvorgangs diente eine periodische Entnahme einer geringen Probenmenge aus dem Versuchsreaktor. Eine Verallgemeinerung der diffusionskontrollierten Modellgleichung ermöglichte die Auswertung des Konzentrationsanstiegs an Bindermetall in der Lösung über die Versuchszeit. Schlussendlich stellen sogenannte Prediction Plots die Wirkung der variablen Parameter (Säure, $\mathrm{H}_{2} \mathrm{O}_{2}$, Temperatur) auf die Geschwindigkeit der Binderlaugung dar.

Ausgehend von den gewonnenen Erkenntnissen finden gegenwärtig Versuche zur Bestimmung des Langzeitverhaltens über mehrere Tage bzw. Wochen statt. Mit diesen lässt sich die Selektivität (Co/WC) genauer beurteilen. Beispielsweise sind Passivierungseffekte und Säuren mit kolloidalem $\mathrm{WO}_{3} \cdot \mathrm{H}_{2} \mathrm{O}$ sichere Anzeichen für einen Angriff auf die Hartphase. Ebenfalls steht dann ausreichend Material für die
Bewertung der Rezyklatqualität nach der Pulverisierung zur Verfügung. Die nächsten Schritte umfassen auch die Klärung der Beständigkeit der üblichen CVD- sowie PVD-Schichten und deren Barrierewirkung auf die Laugungsreaktion.

\subsection{Semidirektes Recycling von Bohrkronen}

Die PDC-Schneider sowie der Verbindungsteil aus Stahl (Abb. 8) werden abgetrennt und gesondert behandelt. Im Fokus der Forschungsarbeiten liegt die Aufarbeitung des hartmetall- und stahlhaltigen Bohrkopfs mit dem primären Ziel, die Wolframphase für die Herstellung neuer Meißel zugänglich $\mathrm{zu}$ machen. Besondere Herausforderungen bestehen im geringen Oberflächen/Volumen-Verhältnis des Bohrkopfschrottes, der Mischung verschiedener Hartphasen sowie der komplexen, korrosionsbeständigen Binderlegierung.

Durch die selektive Auflösung der Bindelegierung soll die Hartphase mit einem möglichst einfachen hydrometallurgischen Aufbau und geringem Energieaufwand als wenig verunreinigter fester Rückstand wiedergewonnen werden. Im Rahmen des Forschungsprojekts wurden unter anderem die folgenden Fragen beantwortet.

1. Welche Lösungen eignen sich für eine selektive Binderlaugung?

Da es sich um eine komplexe Bindelegierung aus Kupfer, Mangan, Nickel und Zink handelt, die ihrem Anforderungsprofil gemäß eine gute Korrosionsbeständigkeit aufweist, ist ihre Auflösung in einem angemessenen Zeitraum nur unter stark oxidierenden Bedingungen möglich (Holleman et al. 2007). Dies konnte in Versuchen nachgewiesen werden, bei denen Proben aus infiltriertem Hartmetall bei $50^{\circ} \mathrm{C}$ und Umgebungsdruck für $4 \mathrm{~h}$ mit unterschiedlichen Laugungsmedien in Kontakt gebracht wurden. Es wurde gezeigt, dass verdünnte Salpetersäurelösungen zu einer raschen Auflösung des Binders führen. Weiters war diesbezüglich auch die Kombination einer Mineralsäure wie Perchlor-, Salzoder Schwefelsäure mit dem Oxidationsmittel Wasserstoffperoxid. Das Einleiten von Sauerstoff sowie die Verwendung von organischen Säuren oder Basen führte $\mathrm{zu}$ keinem starken Angriff. Um eine selektive 
Lösung zu erreichen, muss eine maximale Binderlaugung mit einer minimalen Wolframlöslichkeit kombiniert werden. Während sich zeigte, dass bei den Versuchsbedingungen geringe Mengen an Wolfram in Lösung gingen - maximal $0,2 \mathrm{~g} / \mathrm{l} \mathrm{im}$ Vergleich zu einer maximalen Bindemetallkonzentration von etwa $60 \mathrm{~g} / \mathrm{l}$ -, wurden besonders geringe $\mathrm{W}$-Konzentrationen von weniger als $0,1 \mathrm{~g} / \mathrm{l}$ mit verdünnter Salpetersäure und etwas höhere bei der Zugabe von $\mathrm{H}_{2} \mathrm{O}_{2}$ erreicht (Kerschbaumer et al. 2017a).

2. Kann eine hinreichend reine Wolframphase gewonnen werden?

Nach der Auflösung der infiltrierten Hartmetallproben konnte die wolframhaltige Phase durch Filtration von der Lösung abgetrennt werden. Die Messung mittels Röntgenfluoreszenzanalyse (RFA) ergab geringe Gehalte an Verunreinigungen von weniger als 2 Gew.-\% bei jenen Proben, welche mit der stark oxidierenden $\mathrm{HNO}_{3}$ gelaugt wurden. Maximale Fremdelementgehalte von 13 Gew.-\% wurden bei der Laugung mit Eisenchlorid und Wasserstoffperoxid gemessen (Kerschbaumer et al. 2017a).

3. Wie ist das zeitabhängige Laugungsverhalten der unterschiedlichen Hartphasen?

In den Kinetikuntersuchungen wurden Proben aus infiltriertem W, WC und CTC sowie der Bindelegierung unter variierenden Temperaturen und Konzentrationen mit verdünnter Salpetersäure sowie Lösungen aus Salzsäure und Wasserstoffperoxid gelaugt. Sowohl die Bindelegierung als auch das infiltriertes Wolframkarbid zeigen in $\mathrm{HNO}_{3}$ einen konstanten Anstieg der Bindemetallkonzentrationen. Dies ist damit zu erklären, dass es sich um eine chemisch kontrollierte Reaktion mit Lösungsmittelüberschuss handelt, wonach die Reaktionsgeschwindigkeit unabhängig von der Zeit ist. Im Gegensatz dazu bildet sich bei infiltriertem Wolfram eine feste Produktschicht aus, welche als Diffusionsbarriere gegen die Lösung wirkt. Es kommt zu einer Abschwächung der Reaktionsgeschwindigkeit durch das Anwachsen des Wolframskeletts, wodurch das Vordringen der Säure zur Binderoberfläche erschwert wird. Bei infiltriertem Wolframschmelzkarbid ist ebenfalls eine Verringerung der Binderlaugungsgeschwindigkeit mit der Zeit zu erkennen (Kerschbaumer et al. 2017a).

Wird Salzsäure gemeinsam mit Wasserstoffperoxid als Lösungsmittel verwendet, erfolgt für alle Materialien eine deutliche Reduktion der Laugungseffizienz. Dieser Effekt tritt besonders stark bei Bedingungen auf, bei denen das Oxidationsmittel $\mathrm{H}_{2} \mathrm{O}_{2}$ eine geringe Beständigkeit in der Säure hat, wie bei hohen Temperaturen und $\mathrm{HCl}-$ Konzentrationen (Kerschbaumer et al. 2017b).

Der Verlauf der relativen Kupferkonzentration für die untersuchten Material- und Lösungsmittelkombinationen in Abb. 15 verdeutlicht, dass bei der vollständigen Auflösung des Bohrkopfes mit signifikanten Unterschieden zwischen Schulter- und Körperbereich zu rechnen ist.

In Bezug auf die Wolframlöslichkeit konnte klar und in Übereinstimmung mit der Literatur gezeigt werden, dass das Refraktärmetall in $\mathrm{HNO}_{3}$ eine passivierende Oxidschicht ausbildet, während in $\mathrm{HCl}$ und $\mathrm{H}_{2} \mathrm{O}_{2}$ hohe Wolframkonzentrationen erzielt werden (Lassner und Schubert 1999; Best et al. 1987).

\section{Zusammenfassung}

Durch die hohe wirtschaftliche wie auch strategische Relevanz von Wolfram ist dieses Refraktärmetall der Gruppe der kritischen Rohstoffe zuzuordnen. Das vergleichsweise hohe Verhältnis von österreichischen Importen zur weltweiten W-Produktion von $>1 \%$ unterstreicht zudem dessen Relevanz für Österreichs Ökonomie. Die bedeutendste Endanwendung von Wolfram stellt mit einem Anteil von $54 \%$ (LealAyala et al. 2015) die Sparte der Hartmetalle dar. Dieses Verbundmaterial besteht aus mindestens einer hochschmelzenden, keramischen Phase, in den meisten Fällen Wolframkarbid, und einem metallischen Binder, vorwiegend aus der Eisengruppe. Hinsichtlich der Exporte von WC übertrifft Österreich mit 35,1\% (Liedtke und Schmidt 2014) der weltweit ausgeführten Mengen sogar China und liegt hinsichtlich der global eingeführten Stoffströme von wolframhaltigen Abfällen und Schrotten mit $11 \%$ (Liedtke und Schmidt 2014) unter den drei größten Importeuren. Die Aufarbeitung von WC-haltigen Materialien am Ende ihres Produktle- benszyklus stellt somit einen wichtigen Aspekt für Österreichs Wirtschaft dar.

Prinzipiell stehen für das Recycling dieser Reststoffe direkte, semidirekte oder indirekte Verfahrensvarianten zur Verfügung. Diese Methoden finden seit Langem im industriellen Maßstab Einsatz und arbeiten im direkten Bereich vor allem mit sauberen, sortenreinen Schrotten bzw. mit hohem prozesstechnischen Aufwand in der indirekten Route. Dennoch besteht beispielsweise hinsichtlich eingeschleppter Verunreinigungen bzw. neuartigen Bindersystemen nach wie vor erheblicher Forschungs- und Entwicklungsbedarf, wodurch bestehende Recyclingmethoden hinsichtlich der neuen Herausforderungen zunehmend an ihre Grenzen stoßen. Daher beschäftigt sich das CDLabor für Extraktive Metallurgie von Technologiemetallen unter der Leitung von Priv.-Doz. DI Dr. Stefan Luidold am Lehrstuhl für Nichteisenmetallurgie der Montanuniversität Leoben intensiv mit den Grundlagen des Recyclings von Verbundwerkstoffen, um die Basis für weitere Optimierungen dieser Thematik zu bilden. Im Rahmen der laufenden Studien liegt der Fokus vor allem auf direkten und semidirekten Verfahren zum Recycling von Schneideinsätzen und Bohrkronen. Die Studien zum Zinkprozess gaben Aufschluss über den grundlegenden Ablauf der Desintegration der Hartmetalle im flüssigen Zink, Diffusionsbarrieren und die Auswirkungen von zusätzlichen Binderlegierungselementen. Im Bereich der semidirekten Aufarbeitungstechnologien von WC-Co-Materialen fanden detaillierte Studien zur selektiven Laugung statt, welche unterschiedliche Säuren, Wasserstoffperoxid und Additive in Abhängigkeit der entsprechenden Prozessparameter und den Reaktionsfortschritt in den Vordergrund stellte. Eine besondere Herausforderung beim semidirekten Recycling von Bohrkronen ergibt sich durch das komplexe Bindersystem. Für die aktuellen Arbeiten stehen Kinetikuntersuchungen und das Laugungsverhalten diverser Ausgangsmaterialien sowie die Selektivität der jeweiligen Lösungen im Fokus. Diese Studien sollen einen wesentlichen Beitrag zur gezielten Optimierung von bestehenden Recyclingrouten liefern bzw. die Grundlage für Neuentwicklungen schaffen, um somit die Aufarbeitung von WC-Verbundmaterialen nachhaltig und effizient zu verbessern. 


\section{Ausblick}

Die bisherigen eigenen Forschungstätigkeiten und Recherchen bezüglich des Recyclings von Verbundwerkstoffen mit Wolframkarbid als eine Hauptkomponente weisen darauf hin, dass trotz der Erfahrungen aus der langjährigen industriellen Anwendung die beim Aufschluss ablaufenden chemischen und physikalischen Vorgänge im Detail noch nicht umfassend bekannt sind. Aus diesem Grund konzentrieren sich die weiteren geplanten Untersuchungen unter anderem auf die Vervollständigung dieses Wissens, um eine bestmögliche Ausgangsbasis für die Anpassung des Wolframrecyclings an neue Entwicklungen im Werkstoffbereich (Beschichtungen, Bindersysteme etc.) bzw. für die Erweiterung der nutzbaren Sekun- därrohstoffbasis zu schaffen. Darüber hinaus erfordern auch die nachfolgenden Prozessschritte für die Umsetzung der aufgeschlossenen Schrotte zu marktfähigen oder wiederverwendbaren Rohstoffen ebenso eine Ergänzung der grundlagen- und anwendungsorientierten Kenntnisse für die Erzielung einer optimalen Effizienz der gesamten Recyclingroute. Längerfristig soll das erarbeitete Know-how ebenso für die Entwicklung von Prozessketten für die Wiederverwertung von ähnlichen Schrotten, wie beispielsweise Cermets, Wolfram-Schwermetalllegierungen etc., Anwendung finden.

Danksagung Die Autorinnen und Autoren bedanken sich beim Bundesministerium für Wissenschaft, Forschung und Wirtschaft sowie der Nationalstif- tung für Forschung, Technologie und Entwicklung für die finanzielle Unterstützung.

Funding Open access funding provided by Montanuniversität Leoben.

Open Access Dieser Artikel wird unter der Creative Commons Namensnennung 4.0 International Lizenz (http:// creativecommons.org/licenses/by/4. $0 /$ deed.de) veröffentlicht, welche die Nutzung, Vervielfältigung, Bearbeitung, Verbreitung und Wiedergabe in jeglichem Medium und Format erlaubt, sofern Sie den/die ursprünglichen Autor(en) und die Quelle ordnungsgemäß nennen, einen Link zur Creative Commons Lizenz beifügen und angeben, ob Änderungen vorgenommen wurden.

\section{Literatur}

Adams, N.J., Charrier, T. (1985): Drilling Engineering: A Complete Well Planning Approach. PennWell Books, Tulsa

Ad-hoc Working Group (2010): Critical raw materials for the EU: Report of the Ad-hoc Working Group on defining critical raw materials. https:// nachhaltigwirtschaften.at/resources/e2050 pdf/reports/20100730_critical_raw_materials for_the_eu.pdf?m=1469660947. Gesehen 01 . September 2016

Alkatsev, M.I., Svistunov, N.V., Trotsenko, I.G. (2008): Regeneration of the WC-Co hard alloy with the use of gaseous zinc. Russ. J. Non-ferrous Metals 49 (3):156-159. doi:https://doi.org/ 10.3103/S106782120803005X

Angerer, T., Luidold, S., Antrekowitsch, H. (2011a): Technologien zum Recycling von Hartmetall: Teil 1. World of Metallurgy - ERZMETALI 64:5-14

Angerer, T., Luidold, S., Antrekowitsch, H (2011b): Technologien zum Recycling von Hartmetall: Teil 2. World of Metallurgy - ERZMETALL (64):62-70

Azar, J.J., Robello Samuel, G. (2007): Drilling Engineering. PennWell nontechnical series. Penwell Books, Tulsa

Barnard, P., Starliper, A., Kenworthy, H. (1969): Reclamation of Refractory Carbides from Carbide Materials (US 3595484)

Best, E., Kuhn, P., Kurtz, W., List, H. (1987): Gmelin Handbook of Inorganic Chemistry: W Tungsten. Springer-Verlag Berlin Heidelberg Bock, A. (2017): Raw materials in cemented carbide. Euro Hard Materials Group Meeting 31.05.2017, Reutte

Böhlke, W. (2002): Hartmetall - ein moderne Hochleistungswerkstoff. Materialwissenschaft und Werkstofftechnik 33:575-580

Bose, A. (2011): A perspective on the earliest commercial PM metal-ceramic composite: $\mathrm{Ce}$ mented tungsten carbide. International Journal of Powder Metallurgy 47 (2):31-50

British Geological Survey (2011): Tungsten profile. https://www.bgs.ac.uk/downloads/ 作 SEARCH_TXT=tungsten\&dlBtn=go. Gesehen 09 . Juni 2015

Brookes, K. (2014): Hardmetals recycling and the environment. Metal Powder Report 69
(5):24-30. doi:https://doi.org/10.1016/S00260657(14)70225-5

Brown, T.J., Hobbs, S.F., Idoine, N.E., Mills, A.J. Wrighton, C.E., Raycraft, E.R., Bide, T., Deady, E.A., Rippingale, J., MacKenzie, A.C. (2016): European Mineral Statistics 2010-14. British Geological Survey (BGS), Keyworth, Nottingham

Brown, T.J., Idoine, N.E., Raycraft, E.R., Shaw, R.A., Deady, E.A., Hobbs, S.F., Bide, T. (2017): World Mineral Production 2011-2015. British Geological Survey (BGS), Keyworth, Nottingham Ceratizit S. A. (2015): Wood Working. Gesamtkatalog. http://www.ceratizit.com/uploads/tx extproduct/files/GD_KT_PRO-0425-0515_SDE_ ABS_V1.pdf. Gesehen 02. Juli 2015

Ceratizit Austria S. A. (2016a): Rods \& Performs: Main Catalog. http://www ceratizit.com/ services/downloads/. Gesehen 13. Oktober 2016 Ceratizit S. A. (2016b): Cutting Tools: Main Catalog. http://www.ceratizit.com/services/ downloads/. Gesehen 13. Oktober 2016

De Maindreville, B.C., Gomez, W. (2015): Heavy duty matrix bit (US 8973683 B2)

Deutsche Rohstoffagentur (DERA) in der Bundesanstalt für Geowissenschaften (2017): Rohstoffpreismonitor. https://www.deutscherohstoffagentur.de/SharedDocs/GT_Produkte/ Mineral_Rohstoffe/CPL_alle-genTab_DE.html. Gesehen 28. Juli 2017

DIAPAC (2012): Materials \& Components for PDC Matrix Drill Bits. http://www.diapac.com/ wp/wp-content/uploads/2012/04/down_hole_ broch_1mg.pdf. Gesehen 04. August 2017

Ebner, T. (2016): Konzepte zur Optimierung des Hartmetallrecyclings. Dissertation, Montanuniversität Leoben

Ebner, T., Luidold, S., Antrekowitsch, H., Storf, C., Czettl, C. (2017): Phase formation in $\mathrm{Co}(\mathrm{Ru})$ $\mathrm{Zn}$ diffusion couples. Proceedings of the 19th Planseeseminar:RC11/1-RC11-12

Edtmaier, C., Schiesser, R., Meissl, C., Schubert, W.D., Bock, A., Schoen, A., Zeiler, B. (2005): Selective removal of the cobalt binder in $\mathrm{WC} / \mathrm{Co}$ based hardmetal scraps by acetic acid leaching. Hydrometallurgy 76 (1-2):63-71. doi:https://doi. org/10.1016/j.hydromet.2004.09.002

EMUGE-FRANKEN (2004): Handbuch der Gewindetechnik und Frästechnik: Anwendungen,
Tipps, Tabellen. Publicis Corporate Publishing, Erlangen

Fang, Z.Z., Koopman, M.C., Wang, H. (2014) Cemented Tungsten Carbide Hardmetal-An Introduction. Comprehensive Hard Materials 1:123-137. doi:https://doi.org/10.1016/B978-008-096527-7.00004-0

Gee, M.G., Gant, A.J., Roebuck, B., Mingard, K.P. (2014): Wear of Hardmetals. Comprehensive Hard Materials 1:363-383. doi:https://doi org/10.1016/B978-0-08-096527-7.00012-X

Ghandehari, M.H. (1980): Process for recovering metal carbide powder from cemented carbides (US 4234333)

Gille, G., Meier, A. (2012): Recycling von Refraktärmetallen. In: Thomé-Kozmiensky, K.J. und Goldmann, D.: Recycling und Rohstoffe, Band 5:537-560

Gille, G., Säuberlich, T., Caspers, B. (2012): Zukunftsweisende Entwicklungen bei WC-Hartmetallen: Vorstoffe durch Recycling und Pulverdesign. Pulvermetallurgie in Wissenschaft und Praxis 28:197-224

Gürmen, S., Stopic, S., Friedrich, B. (2005): Recovery of submicron cobalt-powder by acidic leaching of cemented carbide scrap. Proceedings European Metallurgical Conference 2015:1725-1740

Holleman, A. F., Wiberg, E., Wiberg, N. (2007) Lehrbuch der anorganischen Chemie, 102nd edn. de Gruyter, Berlin, New York

Karhumaa, T., Kurkela, M. (2013): Review of the Hardmetal Recycling Market and the Role of the Zinc Process as a Recycling Option. Proceedings of the 18th Planseeseminar:1.138-1.148

Kembaiyan, K., Oldham, T. (2001a): Composition for binder material particularly for drill bit bodies (US 20010002557 Al)

Kembaiyan, K.T., Oldham, T.W. (2001b): Highstrength matrix body (US $6287360 \mathrm{B1}$ )

Kerschbaumer, C., Luidold, S., Wolfe, T., Smith, A. (2017a): Leaching behavior of hard metals used in the drilling industry. Proceedings European Metallurgical Conference 2017:171-185

Kerschbaumer, C., Luidold, S., Wolfe, T, Smith, A. (2017b): Leaching kinetics for semi-direct recycling of drill bits. Proceedings of the 19th Planseeseminar:RC3/1-RC3/12 
Kojima, T., Itoh, H., Sasai, R., Shimizu, T. (2005): Recycling process of WC-Co cermets by hydrothermal treatment. Journal of Materials Science 40 (19):5167-5172

Kopylov, N., Polyakov, B. (1999): Method of disintegrating sintered hard metal carbide bodies and recovering hard metal carbide powder (WO 99/07905 Al)

Kücher, G., Luidold, S. (2017): Semi-direct recycling of cemented carbides in acid media. International Students' Day of Metallurgy, The Austrian Society for Metallurgy and Materials (ASMET), 11.05.2017, Leoben, Austrian Society for Metallurgy and Materials (ASMET)

Kücher, G., Luidold, S., Czettl, C., Storf, C. (Eingereicht, 2016): First evaluation of semidirect recycling methods for the reclamation of cemented carbides based on a literature survey. RevistaMatéria

Kücher, G., Luidold, S., Czettl, C., Storf, C. (2017a): Disclosure of the Kinetic Relations of Semidirect Cemented Carbide Leaching in Acid Media. Rare Metal Technology:159-165. doi:https://doi.org/10.1007/978-3-319-510859_16

Kücher, G., Luidold, S., Czettl, C., Storf, C. (Eingereicht, 2017b): Lixiviation Kinetics of Cobalt from Cemented Carbides. IJRMHM

Kurlov, A.S., Gusev, A.I. (2013): Tungsten Carbides: Structure, Properties and Application in Hardmetals. Springer Series in Materials Science, 184. Springer

Lassner, E., Schubert, W. (1999): Tungsten: Properties, chemistry, technology of the element, alloys, and chemical compounds. Kluwer Academic/Plenum Publishers, New York

Leal-Ayala, D.R., Allwood, J.M., Petavratzi, E., Brown, T.J., Gunn, G. (2015): Mapping the global flow of tungsten to identify key material efficiency and supply security opportunities. Resources, Conservation and Recycling 103:19-28. doi:https://doi.org/10.1016/j.resconrec.2015.07. 003

Liedtke, M., Schmidt, M. (2014): Rohstoffrisikobewertung - Wolfram, 19. Deutsche Rohstoffagentur (DERA) in der Bundesanstalt für Geowissenschaften, Berlin

Lin, J., Lee, S. (1995): Process for recovering tungsten carbide from cemented tungsten carbide scraps by selective electrolysis (US 5384016) Lin, J., Lin, J., Jou, S. (1996): Selective dissolution of the cobalt binder from scraps of cemented tungsten carbide in acids containing additives. Hydrometallurgy 43 (1-3):47-61. doi:https://doi. org/10.1016/0304-386X(96)00023-0 Luidold, S. et al. (2013): Kritische Rohstoffe für die Hochtechnologieanwendung in Österreich: Berichte aus Energie- und Umweltforschung. http://www.nachhaltigwirtschaften.at/ e2050/e2050_pdf/endbericht_1311_kritische_ rohstoffe.pdf. Gesehen 09. August 2016

MacInnis, M.B., Vanderpool, C.D. (1976): Process for the reclamation of uncemented tungsten carbide powders (US 3947555)

McKenna, P.M. (1950): Process for making tungsten monocarbide from tungsten-containing material (US 2529778).

Meyer, H. (1979): Verfahren zur Rückgewinnung von $\mathrm{Ta}(\mathrm{Nb}) \mathrm{C}-\mathrm{WC}$-TiC-Mischungen (DE 2726817 A1)

Oakes, J. (1999): Recycling of Tungsten Materials. 12th Annual General Meeting, Fukuoka Oerlikon Metco (2014): Solutions Flash: Select tailored infiltration materials for PDC drill bits precisely suited for the drilling environment: Select tailored infiltration materials for PDC drill bits precisely suited for the drilling environment. https://www.oerlikon.com/ecomaXL/ files/oerlikon_SF-0020.1_InfiltrationMaterials_ PDCBits_EN.pdf\&download=1. Gesehen 04. August 2017

Paul, R.L., Te Riele, W.A.M., Nicol, M.J. (1985): A novel process for recycling tungsten carbide scrap. International Journal of Mineral Processing 15:41-56

Prakash, L. (2014): Fundamentals and General Applications of Hardmetals. Comprehensive Hard Materials 1:29-90. doi:https://doi.org/10. 1016/B978-0-08-096527-7.00002-7

Ritsko, J.E., MacInnis, M.B., Henson, T.L. (1982): Method of recovering metal carbides (US 4348231) Schedler, W. (1988): Hartmetall für den Praktiker: Aufbau, Herstellung, Eigenschaften und industrielle Anwendung einer modernen Werkstoffgruppe. VDI-Verlag, Düsseldorf

Schiesser, R. (2003): Wertstoffrecycling wolframhältiger Sekundärrohstoffe. Dissertation, TU Wien

Schleinkofer, U., Czettl, C., Michotte, C. (2014): Coating Applications for Cutting Tools. Comprehensive Hard Materials 1:453-469. doi:https:// doi.org/10.1016/B978-0-08-096527-7.00016-7 Schubert, W.D., Lassner, E., Böhlke, W. (2010): Cemented Carbides - A Success Story. http://
www.itia.info/assets/files/Newsletter_2010_06. pdf. Gesehen 14. Januar 2015 Scott, D. (2015): A bit of history: Overcoming early setbacks, PDC bits now drill $90 \%$-plus of worldwide footage. http:/ / www.drillingcontractor. org/a-bit-of-history- overcoming-early-setbackspdc-bits-now-drill-90-plus-of-

worldwide-footage-35932. Gesehen 04. August 2017

Shwayder W.M. (1969): Method of disintegrating sintered hard carbide masses (US 3438730)

Shwayder, WM (1972): Process for Recovering refractory carbide particles from pieces of cemented carbides (US 3635674)

Starck, H.C. (2015): Cast Tungsten Carbide: Technical Data Sheet. https://www.hcstarck. com/cast_tungsten_carbide. Gesehen 04. August 2017

Tikomet Oy (2017): Hartmetallrecycling. http:// www.tikomet.fi/de/hartmetall-recycling/. Gesehen 28. Juli 2017

Uno, T.P., Bird, M.W., Proske, C.A., Dupre, L. (2011): Infiltration Methods for forming drill bits (US 8047260 B2)

Vadasdy, K., Barthaa, L, Szilassy, I., Mikéta, G., Tekula, P. (1986): Verfahren zur Zersetzung und Lösung von Hartmetallschrott durch anodische Oxydation (AT 380495 B)

Vanderpool, C.D. (1983): Electrolytic disintegration of sintered metal carbides (US 4385972)

VAREL Oil \& Gas Drill Bits (2017): Diamond Edge ${ }^{\circledR}$. http://www.vareloilandgas.com/index. $\mathrm{php} / \mathrm{en} /$ fixed-cutter-bits/diamond-edge. Gesehen 10. August 2017

Weil, M., Schubert, W.D. (2013): The Beautiful Colours of Tungsten Oxides. http://www.itia. info/assets/files/newsletters/Newsletter_2013_ 06.pdf. Gesehen 10. Juni 2015

Wolfe, T.A., Jewett, T.J., Singh Gaur, R.P. (2014): Powder Synthesis. Comprehensive Hard Materials 1:185-212. doi:https://doi.org/10.1016/ B978-0-08-096527-7.00006-4

Zeiler, B. (1997): Recycling von Hartmetallschrott, In: Ruthardt, R.: Hartstoffe, Hartstoffschichten, Werkzeuge, Verschleißschutz, Hagener Symposium Pulvermetallurgie 1997, Hagen, 13.-14. 11.1997, Werkstoff-Informationsgesellschaft, 283-303 